\title{
Irisin receptor in osteocytes identified
}

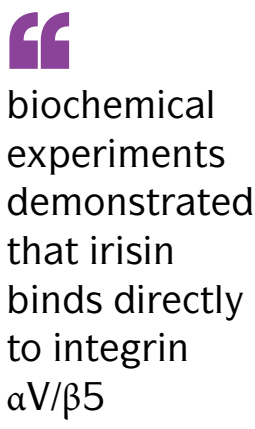

Levels of the myokine irisin increase as a result of exercise, and irisin is known to be involved in many of the beneficial effects of exercise, particularly in adipose tissues, bone and the brain. However, exactly how the skeleton responds to exercise has been unclear, and the receptor for irisin had not been identified. Now, new research published in Cell has demonstrated that the $\alpha \mathrm{V}$ integrins are the receptors for irisin in osteocytes. This new paper also explores some of the effects of irisin in these cells.

"Papers had appeared about effects of irisin on bone and so we began to explore effects in clonal bone cell lines," explains Bruce Spiegelman, corresponding author. "With the help of several bone labs (Clifford Rosen, Lynda Bonewald and Roland Baron) we began to explore this more seriously." The researchers began by demonstrating that irisin treatment increased the expression of sclerostin mRNA in an osteocyte-like cell line called MLO-Y4 (sclerostin regulates bone remodelling). In the same cell line, irisin treatment was also found to

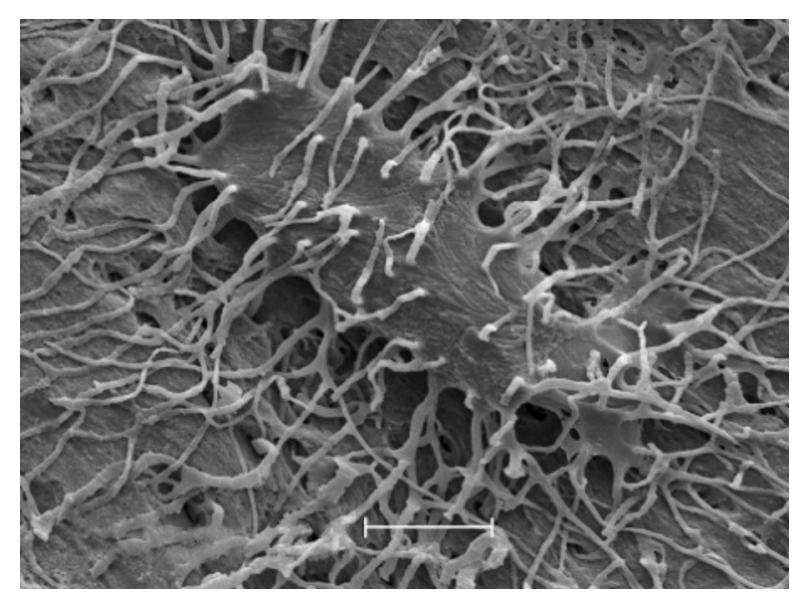

Electron microscopy image of the osteocyte lacunocanalicular network produced by acid etching of resin embedded murine bone. Scale bar represents $5 \mu \mathrm{m}$. Image courtesy of L. F. Bonewald. protect the cells from hydrogen peroxide-induced apoptosis.

Next, the researchers examined the effects of knocking out the gene that encodes fibronectin type III domain-containing protein 5 (FNDC5; the irisin precursor) in mice. Compared with wild-type littermates, the knockout mice had reduced levels of receptor activator of nuclear factor- $k B$ ligand (RANKL), which is involved in osteoclast activation. The knockout mice also had increased femoral trabecular bone mass and increased connectivity density, which suggests that these mice had reduced bone resorption. The researchers also performed ovariectomies in these mice. As expected, the wild-type mice had increased bone resorption and bone loss following ovariectomy. However, similar bone loss was not seen in the knockout mice, which indicates they were resistant to the effects of ovariectomy on bone. Spiegelman and colleagues therefore suggest that FNDC5 (and thus irisin) is required for the effects of ovariectomy on bone, and that irisin also induces bone resorption via effects on osteocytes.

The researchers then conducted a series of experiments designed to identify the irisin receptor. They first performed quantitative proteomics analyses in MLO-Y4 osteocytes, which identified five cell surface proteins that might be receptors for irisin. Of these, only integrin $\beta 1$, which forms obligate heterodimers with a integrins, was known to bind protein ligands.

When ligands bind to these integrin heterodimers, a well described integrin signalling pathway is activated. To test whether irisin could elicit the activation of this pathway, the researchers treated
MLO-Y4 cells with doses of irisin that were as low as $0.1 \mathrm{nM}$. Only the cells treated with irisin displayed activation of the classic integrin signalling pathway. Spiegelman and colleagues therefore conclude that irisin stimulates a pathway of integrin-like signalling. In addition, chemical inhibition of integrins blocked irisin-induced signalling, further supporting the notion that integrins are required for irisin to function in osteocytes.

Next, the investigators performed a binding assay using purified recombinant irisin and a range of commercially available integrin complexes. Of the tested complexes, integrin $\alpha \mathrm{V} / \beta 5$ showed the highest levels of binding. Further biochemical experiments demonstrated that irisin binds directly to integrin $\alpha \mathrm{V} / \beta 5$.

"I think the most important result is the identification of a receptor that responds to physiological doses of irisin, which opens up studies in other tissues as well," says Spiegelman. "Of course, these studies suggest that irisin is an important component of bone health and might be exploited therapeutically in osteoporosis." The researchers are now planning to extend their findings by studying the therapeutic potential of irisin and how inhibiting irisin effects bone biology. "We are also testing irisin in models of other diseases that might benefit, including diseases of neurodegeneration such as amyotrophic lateral sclerosis and Alzheimer disease," concludes Spiegelman.

Claire Greenhill

ORIGINAL ARTICLE Kim, H. et al. Irisin mediate effects of bone and fat via $\mathrm{a} V$ integrin receptors. Cell https://doi.org/10.1016/j.cell.2018.10.025 (2018)

FURTHER READING Perakakis, N. et al. Physiology and role of irisin in glucose homeostasis. Nat. Rev. Endocrinol. 13, 324-337 (2017) 